\title{
Especificidades e importância de modelos matemáticos de qualidade da água
}

\author{
Specificities and importance of mathematical models of water quality
}

\author{
Leandro Fleck ${ }^{1}$ \\ Maria Hermínia Ferreira Tavares ${ }^{2}$ \\ Eduardo Eyng ${ }^{3}$
}

\begin{abstract}
Resumo: Como alternativa aos impactos ambientais provenientes do desenvolvimento econômico várias ferramentas de apoio a gestão pública vêm sendo desenvolvidas, onde os modelos matemáticos de qualidade da água merecem especial destaque por apresentarem ampla aplicação no processo de gerenciamento da qualidade dos recursos hídricos. Neste sentido, o presente trabalho tem como objetivo apresentar uma revisão bibliográfica acerca dos principais fatores relacionados à modelagem matemática da qualidade da água de rios. Ao criar um modelo matemático algumas etapas devem ser consideradas para que as informações geradas sejam confiáveis. Dentre as principais etapas, o processo de calibração e validação do modelo se destaca, pois nessas etapas o modelo é confrontado com os dados experimentais e o poder preditivo do mesmo é confirmado. Em geral, a modelagem matemática quando corretamente utilizada por profissionais capacitados, engloba processos químicos, físicos e biológicos de forma simples e eficiente, justificando assim seu uso no controle da qualidade dos recursos hídricos.
\end{abstract}

Palavras-chave: Modelagem Matemática; Recursos Hídricos; Gestão Pública.

\begin{abstract}
As alternative to environmental impacts from the economic development various tools to support public management have been developed, where the mathematical models of water quality deserve special featured by presenting wide application in the process of quality management of water resources. In this sense, this paper aims to present a bibliographic review on the main factors related to the mathematical modeling of water quality of rivers. By creating a mathematical model some steps must be considered so that the information generated is reliable. Among the steps principals, the calibration and validation of the model stands out because these steps the model is confronted with the experimental data and the predictive power of the same is confirmed. In general, mathematical modeling when properly used by capacitated professionals encompasses process chemical, physical and biological simply and efficiently, thus justifying its use in controlling the quality of water resources.
\end{abstract}

Keywords: Mathematical Modeling; Water Resources; Public Management.

\footnotetext{
${ }^{1}$ Técnologo em Gestão Ambiental; Discente do Programa de Pós-Graduação em Engenharia Agrícola; Departamento de ciências exatas e tecnológicas - Universidade Estadual do Oeste do Paraná.

2 Doutora e Mestre em Física, graduada em Química e Engenharia Química. Professora associada do Departamento de ciências exatas e tecnológicas - Universidade Estadual do Oeste do Paraná.

${ }^{3}$ Doutor e Engenheiro Químico; Docente da UTFPR - Universidade Tecnológica Federal do Paraná, Câmpus Medianeira, Núcleo de Ciências Biológicas e Ambientais.
} 


\section{Introdução}

$\mathrm{Na}$ atualidade, muitas pessoas habitam regiões situadas nas proximidades de córregos e Áreas de Preservação Permanente (APP), promovendo grande impacto ambiental através de ações que deterioram a qualidade da água, principalmente com o descarte de esgotos domésticos sem tratamento prévio (RIBEIRO; ARAÚJO, 2002). Diante disso, muitas tecnologias e programas computacionais surgem como alternativa à resolução dos problemas ambientais (ZHANG et al, 2012), com o objetivo de expressar matematicamente diferentes bioprocessos envolvidos na biodegradação de águas residuais.(MARTIN; AYESA, 2010).

Os modelos matemáticos de qualidade da água são ferramentas eficientes na simulação de mudanças físicas, químicas e biológicas que ocorrem em sistemas aquáticos, contudo, é necessário que as informações provenientes de seu uso sejam corretamente interpretadas para que ações corretivas possam ser desenvolvidas (SONG; KIM, 2009). Segundo Pimpan e Jindal (2009), essas informações podem ser amplamente utilizadas pelos gestores públicos no processo de gestão dos recursos hídricos.

Neste sentido, segundo Cox (2003) os modelos de qualidade da água são amplamente utilizados por órgãos ambientais no controle da poluição causada por empresas em todo o mundo. Assim, o uso da modelagem é em grande parte impulsionada pela legislação vigente em cada país, diferindo em regiões diferentes. Para Ani et al. (2009), a utilização de modelos matemáticos não se restringe somente à simulação de impactos causados por cargas poluentes consideradas comuns, mas são úteis na simulação de impactos causados por poluentes que acidentalmente atingem os cursos hídricos.

De acordo com Rode et al. (2007), os modelos de qualidade da água são cada vez mais utilizados na resolução de problemas complexos envolvidos no monitoramento da qualidade da água, o que implica na crescente necessidade de inserir a modelagem matemática no desenvolvimento dos planos de gestão das bacias hidrográficas. Augusto e Bamigbola (2007), afirmam que os modelos matemáticos desempenham um papel importante na previsão do nível de poluição nas regiões consideradas.

Os principais modelos utilizados atualmente na modelagem da qualidade da água de rios são: QUAL2E, WASP, Mike 11 e QUAL-UFMG. A correta seleção do modelo utilizado na determinação da qualidade da água de determinado curso hídrico dependerá dos dados disponíveis e do tipo de problema a ser considerado (FERRER et al, 2012). Neste sentido, segundo Neuman (2003) a utilização de um modelo inadequado no processo de gestão dos recursos hídricos pode apresentar erros superiores ao erro decorrente da escolha inadequada dos valores dos parâmetros do modelo. O presente trabalho tem como objetivo apresentar uma revisão bibliográfica sobre modelagem matemática aplicada a análise da qualidade da água de rios.

\section{Evolução do uso da modelagem matemática}


Ao longo dos anos várias técnicas quantitativas foram criadas para avaliar o impacto do lançamento de águas residuais nos cursos hídricos. Estas vão desde os conceitos básicos de equilíbrio de massa utilizado em 1912, até o uso atual de softwares de computador na simulação da cinética de decomposição de poluentes lançados sem prévio tratamento no meio natural (COX, 2003). Para Borsuk et al. (2003), os modelos matemáticos de qualidade da água representam tentativas de combinar a compreensão obtida a partir de vários estudos em uma única estrutura preditiva.

Vários modelos têm sido desenvolvidos para vários tipos de cursos hídricos (rios, lagos, reservatórios). Alguns desses modelos têm incluído índices básicos de qualidade da água, como OD (Oxigênio Dissolvido) e DBO (Demanda Bioquímica de Oxigênio), contudo outros modelos mais sofisticados incluem critérios mais elaborados de qualidade da água, como níveis de eutrofização e impactos de toxicidade (FAN et al., 2009).

Nas últimas décadas a percepção ambiental dos atores sociais e do poder público aumentou consideravelmente, elevando o uso dos modelos matemáticos no controle da poluição dos recursos naturais (FITZPATRICK, 2009). Contudo, apesar do progresso observado nos últimos tempos, a credibilidade dos modelos matemáticos para formar a base das decisões das políticas públicas tem sido severamente criticada na literatura. A principal crítica feita é de que a validação de qualquer tipo de modelo com o objetivo de reproduzir um sistema aberto é praticamente impossível (RAMIN et al., 2012).

O modelo de Streeter-Phelps foi o pioneiro para os modelos matemáticos atuais, abordando dois aspectos importantes: o consumo de oxigênio pela oxidação da matéria orgânica e a produção de oxigênio pela reaeração atmosférica. É um modelo determinístico por ter um conjunto de entradas conhecidas e estatístico por não ser alterado em função do tempo (VON SPERLING, 2007).

Desde o surgimento do modelo de Streeter-Phelps, vários modelos foram criados como alternativa a diferentes problemas relacionados à baixa qualidade da água. Todos esses modelos possuem um único objetivo em comum: auxiliar os gerenciadores dos recursos hídricos no processo de tomada de decisões (SEFFRIN, 2001).

Em geral, os modelos matemáticos são agrupados em diferentes categorias de acordo com as principais características relevantes, como: o ambiente modelado, o uso empregado ao modelo, o número de dimensões consideradas, a forma como os processos são descritos, consideração da variabilidade temporal, forma de análise e distribuição dos dados (COX, 2003).

\section{Modelo QUAL2E}

O modelo QUAL2E foi desenvolvido e lançado pela USEPA (United States Environmental Agency) em 1985 (SONG; KIM, 2009). É um modelo utilizado em estado estacionário ou dinâmico, sendo este uma versão mais recente do modelo QUAL-II (COX, 2003). Em estado estacionário, o modelo é utilizado para avaliar o impacto do lançamento de uma carga poluente no corpo receptor. Dinamicamente, o modelo permite determinar os efeitos das variações dos dados 
meteorológicos e das mudanças da concentração de oxigênio dissolvido, sobre a qualidade da água do curso hídrico em análise (PALMIERI; CARVALHO, 2006).

O modelo QUAL2E é abrangente e versátil, podendo simular até 15 constituintes de qualidade da água: oxigênio dissolvido, demanda bioquímica de oxigênio, temperatura, algas, amônia, nitrito, nitrato, nitrogênio orgânico, fósforo orgânico, fósforo dissolvido, coliformes, três substâncias conservativas e uma arbitrária não conservativa (OPPA, 2007).

Segundo Kanapik et al. (2008), a modelagem utilizando o modelo QUAL2E é realizada com base em dois componentes básicos: equações representativas do processo de escoamento, e equações representativas de transporte de massa, que retratam a variação da concentração da variável de qualidade da água em evidência. O modelo utiliza dois mecanismos para o transporte de poluentes: advecção e dispersão. O balanço de massa que o modelo utiliza para calcular as concentrações das variáveis de qualidade de água simuladas é apresentado na Equação 1:

$V \frac{\partial c}{\partial t}=\partial(\underbrace{A x D_{L} \frac{\partial c}{\partial x}}_{\partial \boldsymbol{x}}) d x-\frac{\partial(A x \bar{U} c)}{\partial \boldsymbol{x}} d x+V \frac{d c}{d t} \pm F e$

(1)

Em que:

V refere-se ao volume, $C$ é a concentração da variável, $A_{x}$ é a área da seção transversal de cada elemento computacional, $D_{L}$ é coeficiente de dispersão longitudinal, $U$ é a velocidade média de escoamento e $F_{e}$ refere-se às fontes externas (entradas ou retiradas).

Rezende (2011) avaliou a qualidade da água do Rio Monjolinho (São Carlos- SP), utilizando o modelo matemático de qualidade da água QUAL2E, através do estudo das interações do nitrogênio nas formas amoniacal, nitrito e nitrato e da concentração de fósforo. Constatou-se que o curso hídrico em análise tem a qualidade de suas águas afetada pelo lançamento de cargas difusas e esgotos sem prévio tratamento. O modelo utilizado no estudo se mostrou uma ferramenta útil para o entendimento dos processos bioquímicos de conversão do nitrogênio e fósforo.

\section{Modelo WASP}

O modelo WASP (Water Analysis Simulation Program- Programa de Simulação de Análise de Água), foi desenvolvido para simular os processos de hidrodinâmica e qualidade da água em uma, duas ou três dimensões, objetivando avaliar o destino e transporte de contaminantes convencionais e tóxicos. O modelo WASP tem sido constantemente utilizado em conjunto com o modelo SWMM, principalmente nos Estados Unidos e América Latina (KANNEL et al., 2011).

O modelo WASP7 é uma versão atualizada do modelo WASP, desenvolvido no ano de 1970. É um modelo hidrodinâmico de sistemas aquáticos. Permite que o usuário estude o impacto de diferentes poluentes sobre a qualidade da água de diferentes meios aquáticos. Os processos de advecção, dispersão e carga difusa 
e pontual são modelados. Os constituintes modelados são: nitrogênio, fósforo, oxigênio dissolvido, demanda bioquímica de oxigênio, algas, produtos químicos orgânicos, metais, mercúrio, agentes patogênicos e temperatura (OPPA, 2007). A aplicabilidade do modelo WASP7 é ampla. Pode ser utilizado para avaliar inúmeros impactos causados a rios, córregos, lagos, lagoas, estuários e águas costeiras (KANNEL et al., 2011).

\section{Modelo Mike11}

O modelo Mike11 foi desenvolvido pelo Instituto de Hidráulica Dinamarquês (DHI), sendo amplamente comercializado nos países da Europa. É bastante utilizado por profissionais da Agência Ambiental da Inglaterra e Gales (EA, Environment Agency) que trabalham com prevenções de enchentes, sendo também usado como modelo de qualidade da água, como parte da metodologia do Gerenciamento da Poluição Urbana, onde é empregado para avaliar o impacto de descartes intermitentes em rios e estuários (LEITE, 2004).

Esse modelo fornece uma aproximação versátil e abrangente em hidrodinâmica e modelagem de rio, com amplo campo de aplicabilidade. Os principais usos desse modelo são: (i) análise de risco de inundação; (ii) previsão de inundação em tempo real; (iii) avaliação da qualidade da água em rios, reservatórios e áreas alagadas; (iv) previsão de qualidade de água e rastreamento de poluente; (vi) transporte de sedimentos e morfologia de rios; (vii) integração da água superficial e subterrânea para análise de água (OPPA, 2007).

Segundo Lucas et al. (2010) o modelo Mike11 foi desenvolvido para simular as variações nas descargas e nível da água em rios como resultado da precipitação ocorrida na bacia hidrográfica, e as entradas e saídas das condições de contorno (fronteiras) do rio. Todos os cálculos que o modelo exige para simular uma previsão são feitos automaticamente, utilizando-se um número individual de módulos.

O módulo chuva-vazão é um modelo determinístico, conceitual e simples, representando a fase terrestre do ciclo hidrológico. O módulo hidrodinâmico contém cálculos da diferença finita implícita de fluxos dinâmicos nos rios baseado nas equações de Saint Venant (LUCAS et al., 2010), descritas a seguir:

$$
\frac{\partial h}{\partial t}+u \frac{\partial h}{\partial x}+h \frac{\partial u}{\partial x}=0
$$

$$
\frac{\partial u}{\partial t}+u \frac{\partial u}{\partial x}+g \frac{\partial h}{\partial y}=g\left(S_{0}-S_{f}\right)
$$

\section{(3)}

Onde:

$\mathrm{t}=$ variável independente relativa ao tempo (s);

$\mathrm{x}=$ variável independente relativa à direção do escoamento $(\mathrm{m})$;

$\mathrm{u}=$ velocidade média de escoamento $\left(\mathrm{m} . \mathrm{s}^{-1}\right)$;

$\mathrm{g}=$ aceleração da gravidade $\left(\mathrm{m} . \mathrm{s}^{-2}\right)$;

$\mathrm{h}=$ espessura da lâmina liquida $(\mathrm{m})$;

$\mathrm{S}_{0}=$ declividade média da calha fluvial ou do fundo do canal $\left(\mathrm{m} . \mathrm{m}^{-1}\right)$;

$\mathrm{S}_{\mathrm{f}}=$ declividade da linha de energia $\left(\mathrm{m} \cdot \mathrm{m}^{-1}\right)$. 
O modelo Mike11 é unidimensional e permite a simulação de fluxos dinâmicos em redes ramificadas e em circuitos fechados. Embora o seu esquema considere as condições de fluxo homogêneas dentro do canal, ele possibilita a simulação de fluxo sobre estruturas como represas (LEITE, 2004).

Lucas et al. (2010) ajustaram o modelo hidrodinâmico Mike11 para a vazão da sub-bacia do Rio Piautinga (Sergipe). O principal parâmetro utilizado para calibrar a descarga do rio em estudo foi o coeficiente de rugosidade de Manning, os demais parâmetros utilizados foram o balanço de calor, a estratificação da coluna de água e a percolação profunda. Os resultados obtidos mostraram que houve um bom desempenho do modelo calibrado para a sub-bacia do Rio Piautinga, demonstrando que o modelo ajustado pode ser utilizado para a estimativa da quantidade de água do meio em estudo.

\section{Modelo QUAL-UFMG}

O modelo QUAL-UFMG, desenvolvido para o ambiente computacional da planilha Excel, possibilita a modelagem da qualidade da água de rios, baseando-se nas características do modelo QUAL2-E, desenvolvido pela USEPA. Esse modelo possibilita a simulação rápida e simples do oxigênio dissolvido, demanda bioquímica de oxigênio, nitrogênio total e suas frações, e coliformes (PASSOS, 2012).

Segundo Costa e Teixeira (2010), o modelo QUAL-UFMG é unidimensional, de interface amigável, adequado para rios com vazões baixas e escoamentos em regime permanente, pois essas condições não favorecem o fenômeno de dispersão longitudinal. De acordo com Von Sperling (2007), o modelo QUALUFMG é mais simples que o modelo QUAL2E. As principais simplificações se devem a: integração numérica pelo método de Euler, a desconsideração da dispersão longitudinal e a não inclusão da modelagem de algas e todas as suas inter-relações com os demais constituintes.

A aplicação do modelo QUAL-UFMG, ocorre com base em quatro planilhas básicas (MOURÃO JÚNIOR, 2010), sendo:

$1^{a}$ FórmulasCoefic: planilha que contém todas as fórmulas utilizadas e os valores usuais dos coeficientes das equações utilizadas na modelagem;

$2^{a}$ DiagramaUnifilar: planilha destinada ao usuário para detalhar o diagrama unifilar do rio que está sendo modelado;

$3^{a}$ RioPrincipal: contém a modelagem do rio principal, onde os cálculos são processados e os resultados apresentados;

4a Tributário1: Caso algum tributário do rio principal seja modelado, esta planilha correspondente é utilizada e os resultados são transportados para a planilha RioPrincipal.

De acordo com Mourão Júnior (2010), o modelo QUAL-UFMG assume o escoamento uniforme em condutos livres. Com base nestes pressupostos e na utilização do Coeficiente de Rugosidade, dado por: 
$U=1 / n\left(R_{h}^{2 / 3} i^{1 / 2}\right)$

(4)

Combinado com a Equação da Continuidade:

$U=Q / A$

(5)

Obtém-se a fórmula de Manning, uma das bases do modelo:

$Q=1 / n\left(A R_{h}{ }^{2 / 3} i^{1 / 2}\right)$

(6)

Onde:

$\mathrm{Q}=\operatorname{vazão}\left(\mathrm{m}^{3} \mathrm{~s}^{-1}\right)$;

$A=$ área da seção transversal $\left(\mathrm{m}^{2}\right)$;

$\mathrm{R}_{\mathrm{h}}=$ Raio hidráulico $(\mathrm{m})$;

$\mathrm{i}=$ declividade $(\mathrm{m} / \mathrm{m})$;

$\mathrm{n}=$ Coeficiente de Rugosidade de Manning.

O modelo QUAL-UFMG incorpora os fenômenos envolvidos no balanço de oxigênio dissolvido, modelando-os em condições de anaerobiose, considerando a sedimentação da matéria orgânica, o consumo de oxigênio dissolvido pela nitrificação, as cargas internas sem vazão e as cargas externas. Os resultados fornecidos pela simulação do modelo são apresentados na forma de tabelas e gráficos (PAULA, 2011).

Costa e Teixeira (2010) avaliaram a qualidade das águas do Ribeirão do Ouro, em Araraquara (SP), aplicando o modelo QUAL-UFMG. A partir dos valores de concentração de oxigênio dissolvido e demanda bioquímica de oxigênio, foi possível verificar o atendimento a legislação vigente. Assim, o modelo se mostrou eficiente no estudo, e os resultados podem servir de base para 0 processo de gerenciamento dos recursos hídricos locais.

\section{Considerações gerais acerca da modelagem matemática}

Análises de qualidade da água baseadas em dados obtidos por monitoramento não apresentam a distribuição da carga poluente ao longo do curso hídrico. Essa informação só pode ser obtida quando os modelos matemáticos são utilizados para interpolar os dados avaliados. A principal vantagem dos modelos matemáticos é incluir mecanismos essenciais que ocorrem em condições naturais, diretamente no processo de interpolação (ERTURK, 2010).

Os modelos matemáticos oferecem várias vantagens no controle da poluição dos cursos hídricos. Por meio de modelos é possível gerar cenários futuros, estabelecer planos de gestão, projetar os prováveis impactos ambientais e estimar os custos das medidas a serem tomadas. Assim, os modelos de qualidade da água são usados como ferramentas de apoio a gestão pública por relatar de forma coerente as possíveis condições futuras dos ecossistemas aquáticos e por ser base científica para melhores planos de gestão (ARHONDITSIS et al., 2006). 
Neste sentido, segundo Gevaert et al. (2012) a modelagem é uma ótima ferramenta no processo de gestão das águas residuais urbanas, permitindo avaliar seu impacto no meio natural antes mesmo de seu lançamento. Consequentemente custos podem ser evitados e os recursos podem ser utilizados de forma mais eficiente.

Em geral, os modelos matemáticos de qualidade da água são utilizados para atender objetivos específicos, pois cada modelo atua de forma peculiar na resolução de diferentes problemas ambientais (REICHERT et al, 2001). Desta forma quando se tem como objetivo criar um novo modelo matemático é necessário obter um nível de conhecimento elevado do sistema. A primeira etapa é definir o objetivo do estudo de modelagem. Esta etapa é muitas vezes esquecida e resulta em modelos ruins ou inúteis. A segunda etapa condiz ao levantamento de informações de modelos já existentes e coleta de dados do sistema estudado. Em seguida é necessário decidir sobre a estrutura do modelo. A calibração também é uma etapa importante, onde o modelo é confrontado com os dados experimentais. Por fim, é necessária a validação do modelo para comprovar o poder preditivo do mesmo (NAESSENS et al, 2012). As etapas geralmente necessárias para a construção de um modelo matemático estão resumidas na Figura 1.

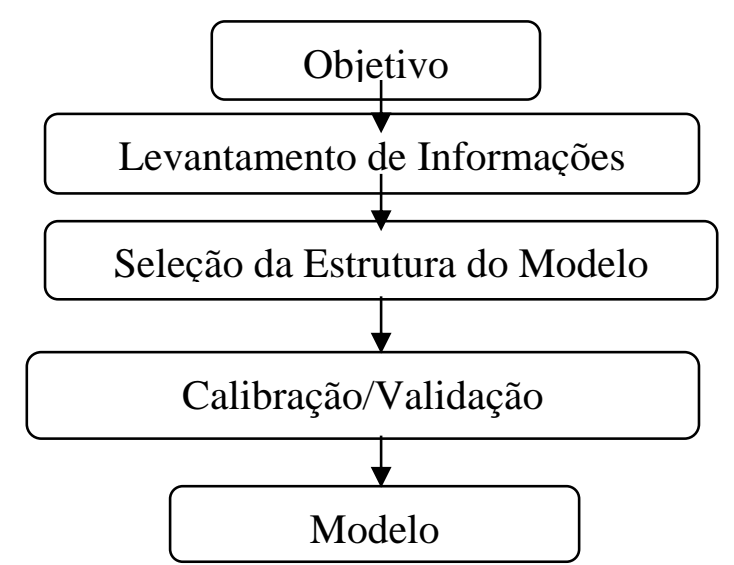

Figura 1- Principais etapas na construção de um modelo matemático.

Fonte: Adaptado de Naessens et al. (2012).

De acordo com Ani et al. (2009), um modelo matemático adequado assegura a estimativa dos parâmetros de interesse com precisão, enquanto o uso de fórmulas já existentes pode levar a estimativas imprecisas. O desenvolvimento de um modelo adequado só será possível com o desenvolvimento correto de todas as etapas apresentadas na Figura 1.

\section{Calibração dos modelos de qualidade da água}

A confiabilidade dos resultados apresentados por um modelo matemático de qualidade da água depende diretamente da forma como o mesmo foi calibrado (HUANG; LIU, 2010). Segundo Fitzpatrick (2009), a calibração é um processo 
pelo qual um modelo e seus coeficientes associados são ajustados para reproduzir da melhor forma possível os dados observados. Segundo Freni et al. (2011) ao trabalhar com modelos complexos, abordagens clássicas de calibração podem se tornar um problema para a confiabilidade dos resultados obtidos.

Durante muito tempo procedimentos de tentativa e erro foram utilizados no processo de calibração de modelos matemáticos de qualidade da água, contudo este método pode não resultar em estimativas confiáveis dos valores dos parâmetros analisados. Além disso, poucos estudos têm sido relatados na literatura sobre a estimativa de parâmetros específicos (MUNAVALLI; KUMAR, 2005).

Nos últimos anos, vários protocolos têm sido propostos para a calibração de modelos matemáticos. Todos estes protocolos salientam a necessidade prévia de definir o objetivo da calibração e verificar a qualidade dos dados para que os resultados sejam satisfatórios (MARTIN; AYESA, 2010).

Em cursos hídricos de baixa vazão, o processo de calibração de modelos simples é dificultado pela escassez de dados disponíveis. Ao lidar com técnicas complexas de modelagem no contexto de dados limitados, abordagens clássicas de calibração podem levar a vários conjuntos de parâmetros igualmente consistentes, tornando difícil a previsão de resultados com elevado grau de confiabilidade (MANNINA; VIVIANI, 2010).

\section{Análise de incerteza e validação de modelos matemáticos}

Segundo Guo e Murphy (2012), a robustez dos resultados modelados é pouco evidenciada na literatura atual. Diante disto Costa e Teixeira (2011) e Larentis (2004), afirmam que existem restrições que devem ser consideradas quando se faz o uso de modelos matemáticos, onde os valores das variáveis de entrada do modelo devem ser ajustados para que os resultados observados representem corretamente a concentração de poluentes existentes no curso hídrico analisado. Assim, diante às imprecisões relacionadas aos resultados obtidos, existe a necessidade de inserir métodos que permitam a avaliação das incertezas nos parâmetros e variáveis de entrada.

Os principais componentes responsáveis pela incerteza nos dados de entrada de um modelo são: erros na estimativa de um dado de entrada, erros na amostragem, erros na medição, calibração ou análise de laboratório, erros na transcrição ou transferência de resultados de análises ou medições, e erros na estimativa de dados de entrada futuros (VON SPERLING, 2007).

O processo de validação de um modelo matemático é realizado para comprovar que os coeficientes adotados nas etapas anteriores apresentam uma precisão satisfatória. Neste sentido Abaurrea et al. (2011), afirma que o processo de validação tem como principal objetivo verificar se os pressupostos do modelo estão corretos, pois do contrário, as conclusões obtidas não seriam aceitas.

Segundo Fitzpatrick (2009) a validação é um processo pelo qual um código de computador e seus algoritmos associados são verificados para garantir que 
matematicamente estão corretos e, além disso, é um meio de observar se os balanços de massas são mantidos ao longo do tempo.

No processo de construção de modelos matemáticos é necessário que ocorra também a análise de sensibilidade do modelo, onde é possível verificar o quanto as variáveis principais variam de comportamento considerando uma variação em seus parâmetros (SAUZEN; SAUZEN, 2010). Segundo Vanni et al. (2009), os parâmetros utilizados em um modelo variam no mundo real, logo, se faz necessário avaliar o impacto destas variações nos resultados encontrados.

Existem três tipos principais de análise de sensibilidade: univariada, multivariada e análise de Monte Carlo. No primeiro caso, varia-se somente um parâmetro por vez, já no segundo, a variação de mais de um parâmetro é feita simultaneamente. A análise de Monte Carlo varia todos os parâmetros ao mesmo tempo, realizando centenas de simulações com as possíveis combinações de valores. Enquanto as primeiras análises citadas nos mostram a sensibilidade do modelo a parâmetros específicos, a última nos mostra a robustez global do modelo.

\section{Considerações finais}

Como consequência do desenvolvimento econômico atual, os impactos causados ao meio ambiente tornaram-se uma das maiores preocupações do novo século. Diante disso, para auxiliar na gestão e proteção dos recursos hídricos é imprescindível a utilização de ferramentas que possibilitem a análise da qualidade da água dos mesmos. Os modelos matemáticos de qualidade da água são utilizados com esses fins, possibilitando estimar o potencial de autodepuração dos recursos hídricos e principalmente, ser base concreta no processo de tomada de decisões.

Uma das vantagens dos modelos matemáticos de qualidade de água é ter uma resposta rápida do comportamento do curso hídrico para diversas condições e variáveis, e a partir dessas informações desenvolver projetos com base nas respostas obtidas com a aplicação do modelo. Exemplos destas aplicações é a melhoria de projetos de estruturas hidráulicas, e controle do lançamento de efluentes em corpos receptores de acordo com os padrões definidos pela legislação vigente (Resolução CONAMA 357/2005).

Por fim pode-se inferir que os modelos matemáticos de qualidade da água quando corretamente utilizados, possuem a capacidade de englobar processos químicos, físicos e biológicos de forma simples e eficiente, justificando assim seu uso. Contudo, para que o modelo se adéqüe corretamente ao sistema estudado é necessário que os objetivos estejam claros e principalmente, que análise das informações seja feita por profissionais devidamente qualificados.

\section{Referências}


ABAURREA, Jesús; ASÍN, Jesús; CEBRIÁN, Ana. C; GARCIA-VERA, Miguel. A. Trend analysis of water quality series based on regression models with correlated errors. Journal of Hydrology. v. 400, n.3-4, p.341-352, nov. 2011.

ANI, Elisabeta. Cristina; WALLIS, Steve; KRASLAWSKI, Andrzej; AGACHI, Paul Serban. Development, calibration and evaluation of two mathematical models for pollutant transport in a small river. Environmental Modelling \& Software. v.24, n.1, p.1139-1152, out. 2009.

ARHONDITSIS, George; ADAMS, Barbara; NIELSEN, Leah; STOW, Craig; RECKHOW, Kenneth. Evaluation of the Current State of Mechanistic Aquatic Biogeochemical Modeling: Citation Analysis and Future Perspectives. Environmental Science Technology. v.40, n.1, p.6547-6554, set. 2006.

AUGUSTO, F. B; BAMIGBOLA, O. M. Numerical treatment of the mathematical models for water pollution. Journal of Mathematics and Statistics. v.3, n.4, p.172-180, 2007.

BORSUK, Mark; STOW, Craig; RECKHOW, Kenneth. Ecological Modelling. v. 173, n.1, p.219-239, abr. 2003.

COSTA, Daniel Jadyr Leite; TEIXEIRA, Denilson. Análise de incerteza em um modelo matemático de qualidade da água aplicado ao Ribeirão do Ouro, Araraquara, SP, Brasil. Revista Ambiente e Água. v.6, n.2, p.232-245, 2011.

COX, B. A. A review of currently available in-stream water-quality models and their applicability for simulating dissolved oxygen in lowland rivers. Science of The Total Environment. v.314-316, n.1, p.335-377, out. 2003.

ERTURK, A; GUREL, M; EKDAL, A; TAVSAN, C; UGURLUOGLU, A; SEKER, D. Z; TANIK, A; OZTURK, I. Water quality assessment and meta model development in Melen watershed - Turkey. Journal of Environmental Management. v.91, n.7,p.1526-1545, mar. 2010.

FAN, Chihhao; KO, Chun-Han.; WANG, Wei-Shen. An innovative modeling approach using Qual2K and HEC-RAS integration to assess the impact of tidal effect on River Water quality simulation. Journal of Environmental Management, v.90, n.5, p.1824-1832, abr. 2009.

FERRER, J; PÉREZ-MARTÍN, M. A; JIMÉNEZ, S; TEODORO, T; ANDREU, J. GISbased models for water quantity and quality assessment in the Júcar River Basin, Spain, including climate change effects. Science of The Total Environment. v.440, n.1, p. 42-59, mar. 2012.

FITZPATRICK, James. Assessing skill of estuarine and coastal eutrophication models for water quality managers. Journal of Marine Systems. v.76, n.1-2, p.195-211, fev. 2009.

FRENI, Gabriele; MANNINA, Giorgio; VIVIANI, Gaspare. Assessment of the integrated urban water quality model complexity through identifiability analysis. Water Research. v.45, n.1, p.37-50, jan. 2011.

GEVAERT, Veerle; VERDONCK, Frederik; BAETS, Bernard. A scenario analysis for reducing organic priority pollutants in receiving water using integrated dynamic 
urban fate models. Science of The Total Environment. v.432, n.1, p.422-431, agos. 2012.

GUO, M; MURPHY, R. J. LCA data quality: Sensitivity and uncertainty analysis. Science of The Total Environment. v.435-436, n.1, p.230-243, out. 2012.

HUANG, Y; LIU, L. Multiobjective Multiobjective Water Quality Model Calibration Using a Hybrid Genetic Algorithm and Neural Network-Based Approach. Journal of environmental engineering. v.136, n.10, p. 1020-1031, out. 2010.

KANAPIK, H. G.; FRANÇA, M. S.; FERNANDES, C. V. S.; MASINI, L. S.; MARIN, M. C. F. C.; PORTO, M. F. A. Análise crítica da calibração do modelo de qualidade de água QUAL2E - estudo de caso da bacia do Alto Iguaçu. Revista de Gestão de Água da América Latina, n.2, v.5, p.25-37, 2008.

KANNEL, P. R.; KANNEL, S. R.; LEE, S.; LEE, Y.; GAN, T. Y. A review of public domain water quality models for simulating dissolved oxygen in rivers and streams. Environmental Modeling \& Assessment, n.2, v.16, p.183-204, 2011.

LEITE, E. B. Simulação do lançamento de esgotos domésticos em rios usando um modelo de qualidade da água, SisBAHIA. 2004. 86f. Dissertação (Mestrado em Saúde Pública) -Escola Nacional de Saúde Pública, Rio de Janeiro, 2004.

LARENTIS, Dante Gama. Modelagem matemática da qualidade da água de grandes bacias: Sistema Taquari-Antas- RS. 2004. 138 f. Dissertação (Mestrado em Recursos Hídricos e Saneamento Ambiental).- Universidade Federal do Rio Grande do Sul, Porto Alegre, 2004.

LUCAS, A. A. T.; AGUIAR NETTO, A. O.; FOlEGATTI, M. V.; FERREIRA, R. A. Calibração do modelo hidrodinámico MIKE11 para a sub-bacia hidrográfica do rio Piauitinga, Sergipe, Brasil. Revista Ambiente \& Água, n.3, v.5, p. 195-207, 2010.

MANNINA, Giorgio; VIVIANE, Gaspare. Water quality modelling for ephemeral rivers: Model development and parameter assessment. Journal of Hydrology. v.393, n.3-4, p.186-196, nov. 2010.

MARTIN, C; AYESA, E. An Integrated Monte Carlo Methodology for the calibration of water quality models. Ecological Modelling. v. 221, n.22, p.2656-2667, nov. 2010.

MOURÃO JÚNIOR, P. R. Aplicação do modelo de autodepuração de qualidade das águas QUAL-UFMG. 2010. 144f. Dissertação (Mestrado Sustentabilidade Sócio-Econômica e Ambiental)- Universidade Federal de Ouro Preto, Ouro Preto, 2010.

MUNAVALLI, G. R; KUMAR, M. S. M. Water quality parameter estimation in a distribution system under dynamic state. Water Research. v.39, n.18, p.42874298, nov. 2005.

NAESSENS, W; MAERE, T; NOPENS, I. Critical review of membrane bioreactor models - Part 1: Biokinetic and filtration models. Bioresource Technology. v.122, n.1, p. 95-106, out. 2012. 
NEUMAN, S. P. Maximum likelihood Bayesian averaging of uncertain model predictions. Stochastic Environmental Research and Risk Assessment. v.17, n.1, p. 291-305, out. 2003.

OPPA, L. F. Utilização de modelo matemático de qualidade da água para análise de alternativas de enquadramento do Rio Vacacaí Mirim. 2007. 129 f. Dissertação (Mestrado em Engenharia Civil) - Universidade Federal de Santa Maria, Santa Maria, 2007.

PALMIERI, V.; CARVALHO, R. J. Qual2e model for the Corumbataí River. Ecological Modelling, n.1-2, v.198, p.269-275, 2006.

PASSOS, R. L. Seleção de eficiências de tratamento de esgotos a partir da utilização combinada de modelo de qualidade da água e de técnica metaheurística de otimização. 2012. 58f. Trabalho de Conclusão de Curso (Engenharia Ambiental) - Universidade Federal do Espírito Santo, Vitoria, 2012.

PAULA, L. M. Avaliação da qualidade da água e autodepuração do Rio Jordão, Araguari (MG). 2011. 196 f. Dissertação (Mestrado em Engenharia Civil) - Universidade Federal de Uberlândia, Uberlândia, 2011.

PIMPAN, P; JINDAL, R. Mathematical modeling of cadmium removal in free water surface constructed wetlands. Journal of Hazardous Materials. v.163, n. 2-3, p. 1322-1331, abr. 2009.

RAMIN, Maryan; LABENCKI, Tanya; BOYD, Duncan; TROLLE, Denis; ARHONDITSIS, George. A Bayesian synthesis of predictions from different models for setting water quality criteria. Ecological Modelling. v. 242, n.1, p.127-145, set. 2012.

REICHERT, $P$; BORCHARDT, $D$; HENZE, $M$; RAUCH, $W$; SHANAHAN, $P$; SOMLYÓDY, L; VANROLLEGHEM, P. A. River water quality Model. Scientific and Technical Report. v. 12, 2001.

REZENDE, G. R. Aplicação do modelo matemático de qualidade da agua QUAL2E para o estudo do comportamento dos nutrientes nitrogênio e fósforo no Rio Monjolinho, São Carlos-SP. XIX Simposio Brasileiro de Recursos Hídricos. Maceió, 2011.

RIBEIRO, César; ARAÚJO, Moacyr. Mathematical modelling as a management tool for water quality control of the tropical Beberibe estuary, NE Brazil. Hydrobiologia. v.475, n.1, p. 229-237, 2002.

RODE, Michael; SUHR, Ursula; WRIEDT, Gunter. Multi-objective calibration of a river water quality model-Information content of calibration data. Ecological Modelling. v.204, n.1-2, p.129-142, mai. 2007.

SAUZEN, A.; SAUZEN, P. S. Aplicação de uma metodologia para análise da sensibilidade do modelo dinâmico para uma tubulação-separador sob golfadas. Sociedade Brasileira de Matemática Aplicada e Computacional, v.11, n.3, p. 245-256, nov. 2010.

SEFFRIN, Gilson Fernando Ferreira. Simulação atual e previsão futura da qualidade das águas do rio Ibicuí utilizando o modelo QUAL2E. 2001. $110 f$. 
Dissertação (Mestrado em Engenharia Civil) - Universidade Federal de Santa Maria, Santa Maria, 2001.

SONG, Tao; KIM, Kyehyun. Development of a water quality loading index based on water quality modeling. Journal of Environmental Management. v.90, n.3, p. 1534-1543, mar. 2009.

VANNI, Tazzio; LUZ, Paula Mendez; RIBEIRO, Rodrigo Antonini; POLANCZYK, Carisi. Avaliação econômica em saúde: aplicações em doenças infecciosas. Caderno de Saúde Pública, Rio de Janeiro, v.25, n.12, p. 2543-2552, dez. 2009.

VON SPERLING, Marcos. Estudos e Modelagem da Qualidade da Água. 1aed. Departamento de Engenharia Sanitária e Ambiental. Minas Gerais: 2007. 588 p.

ZHANG, Ruibin; QIAN, Xin; LI, Huiming; YUAN, Xingcheng; YE, Rui. Selection of optimal river water quality improvement programs using QUAL2K: A case study of Taihu Lake Basin, China. Science of The Total Environment. v.431, n.1, p. 278-285, agos. 2012.

Recebido em: 27/02/2013

Aceito em: 17/06/2013 